\title{
ON THE COMBINATORIAL PROPERTIES OF BLACKWELL SPACES
}

\author{
JAKUB JASIŃSKI
}

\begin{abstract}
Under MA $+\neg \mathrm{CH}$ (Martin's Axiom and negation of the Continuum Hypothesis) we prove that the intersection of a Blackwell space with the analytic set and the Cartesian product of a Blackwell space and a Borel set do not need to be Blackwell spaces.
\end{abstract}

1. Introduction. In this paper we present a few examples illustrating the singular behaviour of Blackwell spaces. They shall provide (under $\mathrm{MA}+\neg \mathrm{CH}$ ) the negative answers to questions P4, P6, P7 and P8 raised by K. P. S. Bhaskara Rao and B. V. Rao in [1]. The first two were originally answered by W. Bzyl and J. Jasiński in [4]. Here we give a slight generalization of their result (Proposition 1).

On the other hand, W. Bzyl in [3], using the idea presented in [9], proved that questions P4, P6, P7 and a weaker version of P8 have positive answers when restricted to Blackwell spaces with totaly imperfect complement in some analytic set.

Let us recall the main definitions. For a metric space $X$ by $\Sigma_{\alpha} X$, we denote the additive Baire classes of Borel subsets of $X$, and by $\mathscr{B}(X)$ we denote a $\sigma$-algebra of all Borel subsets of $X$. A Borel measurable mapping $f: X \rightarrow Y$, where $Y$ is a metric space, is called a class $\Sigma_{\alpha}$ if, for all open subsets $U \subset Y, f^{-1}(U) \in \Sigma_{\alpha}(X)$.

A $\sigma$-algebra of subsets of $X$ is called separable if it is countably generated (c.g.) and separates the points of $X$. Let $X$ be a separable metric space. $X$ is called a Blackwell space if $\mathscr{B}(X)$ does not contain a proper separable sub- $\sigma$-algebra. $X$ is called a strongly Blackwell space if any two c.g. sub- $\sigma$-algebras of $\mathscr{B}(X)$ with the same atoms coincide. It is clear that a strongly Blackwell space is a Blackwell space. If $A$ is an analytic subset of a Polish space, then $A$ is strongly Blackwell. For this and for other results on Blackwell spaces see K. P. S. Bhaskara Rao and B. V. Rao [1].

2. Basic lemmas. We shall often refer to the well-known result of Silver:

LEMMA. 1. (MA) If $Z$ is a separable metric space and $|Z|<2^{\omega}$, then $\mathscr{B}(Z)=\mathscr{P}(Z)$.

For the proof see $[8$, pp. 162,163$]$. As pointed out by K. P. S. Bhaskara Rao and B. V. Rao [1, p. 15], Lemma 1 implies the following

LeMma 2. (MA) If $Z$ is a separable metric space with $|Z|<2^{\omega}$, then $Z$ is strongly Blackwell.

Received by the editors April 25, 1984.

1980 Mathematics Subject Classification. Primary 28A05; Secondary 04A15.

Key words and phrases. Blackwell spaces, Borel sets, analytic sets. 
Lemma 3. If $Y$ is a Blackwell space and B is a Borel subset of the Polish space, then $Y \cup B$ is a Blackwell space.

For the proof see $\left[1\right.$, p. $\left.28,2^{0}\right]$.

3. Main propositions. In this section, $X$ will denote a Polish space.

Proposition 1. (MA) Let $B \in \mathscr{B}(X)$ be of cardinality $2^{\omega}$ and let $Z$ be an uncountable separable metric space of cardinality less than $2^{\omega}$. If $Z \cap B=\varnothing$, then $Z \cup B$ is a Blackwell space which is not strongly Blackwell.

Proof. By Lemma 2, $Z$ is a Blackwell space, so, by Lemma 3, $B \cup Z$ is also a Blackwell space.

W. Bzyl and J. Jasiński in [4] proved that there exists a Borel set $B_{1} \in \mathscr{B}\left(\mathbf{R}^{2}\right)$ and $Z_{1} \subseteq \mathbf{R}$ with $\left|Z_{1}\right|=\omega_{1}$ such that $B_{1} \cup Z_{1}$ is not a strongly Blackwell space. Let $f$ : $B \rightarrow B_{1}$ be a Borel isomorphism (see [6, p. 450, Theorem 2]) and let

$$
g: Z \underset{\text { onto }}{\rightarrow} Z_{1} \text {. }
$$

By Lemma 1 a mapping $h: B \cup Z \rightarrow B_{1} \cup Z_{1}$, defined by

$$
h(x)= \begin{cases}f(x) & \text { for } x \in B, \\ g(x) & \text { for } x \in Z,\end{cases}
$$

is Borel measurable; hence, $B \cup Z$ is not strongly Blackwell.

A certain part of the next proposition does not require MA so we formulate it separately as

Lemma 4. Let $Y \subseteq X$ be a Blackwell (strongly Blackwell) space and let $Z \subseteq X \backslash Y$. If, for every Borel set $B \in \mathscr{B}(X), B \cap Y=\varnothing$ implies $|B \cap Z| \leqslant \omega$, then $Y \cup Z$ is a Blackwell (strongly Blackwell) space. ${ }^{1}$

Proof. We give a proof in case $Y$ is a strongly Blackwell space. Let $\mathscr{C} \subseteq \mathscr{D} \subseteq$ $\mathscr{B}(Y \cup Z)$ be c.g. $\sigma$-algebras with the same atoms and let $D \in \mathscr{D}$. By [1, p. 23, Proposition $8(5)$ ] it suffices to show that $D \in \mathscr{C}$. Since $Y$ is strongly Blackwell, $\mathscr{C} \uparrow_{Y}=\mathscr{D} \uparrow_{Y}$, so there is a set $C \in \mathscr{C}$ such that

$$
C \cap Y=D \cap Y \text {. }
$$

Let $C^{\prime}, D^{\prime} \in \mathscr{B}(X)$ be such that $C^{\prime} \cap(Y \cup Z)=C$ and $D^{\prime} \cap(Y \cup Z)=D$. By (1) the symmetric difference $D^{\prime} \Delta C^{\prime} \subseteq X \backslash Y$; hence, $\left|\left(D^{\prime} \Delta C^{\prime}\right) \cap Z\right| \leqslant \omega$ and $|D \Delta C|$ $\leqslant \omega$, so $D=C \Delta(C \Delta D) \in \mathscr{C}$.

Recall that whenever $A \subseteq X$ is an analytic non-Borel set, then there exist nonempty Borel sets $C_{\alpha}, \alpha<\omega_{1}$, such that each Borel set $B \in \mathscr{B}(X)$ disjoint with $A$ is covered by countably many $C_{\alpha}$ 's. The sets $C_{\alpha}$ are called the constituents of a coanalytic set $X \backslash A$ (see [6, p. 499]).

Disjoint sets $X_{1}, X_{2} \subseteq X$ are called Borel-separable if there is a Borel set $B \in \mathscr{B}(X)$ such that $X_{1} \subseteq B$ and $X_{2} \subseteq X \backslash B$.

\footnotetext{
${ }^{1}$ This lemma has been obtained independently by R. M. Shorttnnn and K. P. S. Bhaskara Rao.
} 
Proposition 2. (MA) Let $A \subseteq X$ be an analytic non-Borel set and let $\left\{C_{\alpha}\right\}_{\alpha<\omega_{1}}$ be the constituents of $X \backslash A$. Whenever $Z \subseteq X, \omega<|Z|<2^{\omega}$ and $A \cap Z=\varnothing$, then $A \cup Z$ is not a Blackwell space iff there is an $\alpha_{0}<\omega_{1}$ such that $\left|C_{\alpha_{0}} \cap Z\right|>\omega$ and $\left|\left\{\alpha: C_{\alpha} \backslash Z \neq \varnothing\right\}\right|=\omega_{1}$.

Proof. "If" part. Let $Z_{1} \subseteq Z \cap C_{\alpha_{0}}$ be of cardinality $\omega_{1}$. Since

$$
\left|\left\{\alpha<\omega_{1}: C_{\alpha} \backslash Z \neq \varnothing\right\}\right|=\omega_{1},
$$

there is a mapping $g: Z_{1} \stackrel{1-1}{\rightarrow} X \backslash(A \cup Z)$ such that $g\left(Z_{1}\right)$ and $A$ are disjoint non-Borel-separable.

Define $f: A \cup Z \stackrel{1-1}{\rightarrow} X$

$$
f(x)= \begin{cases}x & \text { for } x \in(A \cup Z) \backslash Z_{1}, \\ g(x) & \text { for } x \in Z_{1} .\end{cases}
$$

By Lemma $1, f$ is Borel measurable, but $f\left(Z_{1}\right)=g\left(Z_{1}\right) \notin \mathscr{B}(f(A \cup Z))$, so $A \cup Z$ is not a Blackwell space (see [1 p. 22, Proposition 7(2)]).

"Only if" part. Suppose $\left|\left\{\alpha: C_{\alpha} \backslash Z \neq \varnothing\right\}\right|<\omega_{1}$. In this case there is an $\alpha<\omega_{1}$ such that $Z \cup A=Z \cup\left(\cup_{\beta>\alpha} C_{\beta}\right) \cup A$ is Borel, so, by Lemmas 2 and 3, $A \cup Z$ is a Blackwell space.

In case, for every $\alpha<\omega_{1},\left|C_{\alpha} \cap Z\right|<\omega_{1}$, then, for every Borel set $B \in \mathscr{B}(X)$, $B \cap A=\varnothing$ implies $|B \cap Z| \leqslant \omega$, so, by Lemma $4, Z \cup A$ is a Blackwell space.

Corollary 1. (MA) Let $A$ and $Z$ be as in Proposition 2. If $A$ and $Z$ are Borel separable, then $A \cup Z$ is not a Blackwell space.

COROllary 2. $(M A+\neg C H)$ There exists a Blackwell space $Y$ and an analytic set $A \subset X$ such that $Y \cap A$ is not a Blackwell space.

Proof. Let $Y=B \cup Z$ where $B \in \mathscr{B}(X),|B|=2^{\omega}, Z \subset X, \omega<|Z|<2^{\omega}$ and $B \cap Z=\varnothing$. By Lemmas 2 and 3, $Y$ is a Blackwell space. Let $A_{1} \subset B$ be an analytic non-Borel set and take $A=A_{1} \cup(X \backslash B) . A \cap Y=A_{1} \cup Z$ which is not Blackwell by Corollary 1.

Proposition 3. (MA) If $Z \subset X$ and $\omega<|Z|<2^{\omega}$, then $Z \times B$ is not a Blackwell space, where $B$ is an uncountable Borel subset of some Polish space.

We shall precede the proof with two lemmas. The first one follows by Lemma 1 from [2, Theorem 3].

LEMMA 5. (MA) Let $\mathscr{N}$ be a set of irrational numbers. If $Z \subseteq \mathscr{N}$ with $|Z|<2^{\omega}$, then $B \in \mathscr{B}(Z \times \mathscr{N})$ iff there is an $\alpha<\omega_{1}$ such that for every $z \in Z$ the section $B_{z}=\{y$ : $(z, y) \in B\} \in \Sigma_{\alpha}(\mathscr{N})$.

\section{Lemma 5 implies the following}

Lemma 6. (MA) Let $Z$ and $B$ be as in Proposition 3. $A$ mapping $h: Z \times B \rightarrow Y$, where $Y$ is a separable metric space, is Borel measurable iff there is an $\alpha<\omega_{1}$ such that for every $z \in Z$ a restricted mapping $h \uparrow\{z\} \times B$ is of class $\Sigma_{\alpha}$. 
Proof. Apply the isomorphism theorem [6, p. 450, Corollary 1c].

Proof of Proposition 3. By the well-known theorem of Hausdorff [5], $\mathscr{N}$ can be decomposed into $\omega_{1}$ disjoint uncountable sets of class $\Sigma_{3}(\mathscr{N}), \mathscr{N}=\bigcup_{\alpha<\omega_{1}} E_{\alpha}$.

Let $Z_{1} \subset Z$ be of cardinality $\omega_{1}$. By Lemma $1, Z_{1} \times B \in \mathscr{B}(Z \times B)$, so it suffices to prove that $Z_{1} \times B$ is not a Blackwell space (see [1, p. 28, $\left.1^{0}\right]$ ). Let $Z_{1}=\left\{z_{\alpha}: \alpha<\right.$ $\left.\omega_{1}\right\}$. There is a $\gamma<\omega_{1}$ such that for each $\alpha<\omega_{1}$ there is a Borel measurable function $f_{\alpha}: B \stackrel{1-1}{\rightarrow} E_{\alpha}$ of class $\Sigma_{\gamma}$ (see [6, p. 450, Theorem 2]). By Lemma 6, a mapping $h: Z_{1} \times \mathscr{N} \rightarrow \mathscr{N}$ defined by $h\left(z_{\alpha}, x\right)=f_{\alpha}(x)$ is Borel measurable, but the inverse mapping is not, since $Z_{1} \times B$ is not Borel [6, p. 489, Theorem 1]. Hence, by $\left[1\right.$, p. 22, Proposition 7 (2)], $Z_{1} \times B$ is not a Blackwell space.

\section{REFERENCES}

1. K. P. S. Bhaskara Rao and B.V. Rao, Borel spaces, Dissertationes Math. (Rozprawy Mat.) 190 (1981), $1-63$

2. R. H. Bing, W. W. Bledsoe and R. D. Mauldin, Sets generated by rectangles, Pacific J. Math. 51 (1974), 27-36.

3. W. Bzyl, On the analytic dense sets, preprint, 1984.

4. W. Bzyl and J. Jasiński, A note on Blackwell spaces, Bull. Acad. Polon. Sci. 31 (1983), 215-217.

5. F. Hausdorff, Summen von $\mathscr{S}_{1}$ Mengen, Fund. Math. 26 (1936), 248.

6. K. Kuratowski, Topologv I, Academic Press, New York: PWN, Warsaw, 1966.

7. E. Marczewski, Characteristic function of the sequence of sets and some of its applications, Fund. Math. 31 (1938), 207-223.

8. D. A. Martin and R. M. Solovay, Internal Cohen extensions, Ann. Math. Logic 2 (1970), 143-178.

9. R. M. Shortt, Borel dense Blackwell spaces are strongly Blackwell, preprint, 1982.

Institute of Mathematics, University of Gdansk, Ul. Wita Stwosza 57, 80-952 Gdansk, POI.AND 